\title{
A six-gene expression signature related to angiolymphatic invasion is associated with poor survival in laryngeal squamous cell carcinoma
}

\author{
Karl Metzger ${ }^{1}$ (1) Julius Moratin ${ }^{1} \cdot$ Kolja Freier $^{2} \cdot$ Jürgen Hoffmann ${ }^{1} \cdot$ Karim Zaoui $^{3} \cdot$ Michaela Plath $^{3}$. \\ Fabian Stögbauer ${ }^{4}$. Christian Freudlsperger ${ }^{1}$. Jochen Hess ${ }^{5}$. Dominik Horn ${ }^{2}$
}

Received: 6 May 2020 / Accepted: 14 July 2020 / Published online: 20 July 2020

(c) The Author(s) 2020, corrected publication 2021

\begin{abstract}
Purpose Angiolymphatic invasion serves as a histopathological risk factor for unfavorable survival in head and neck squamous cell carinoma. The aim of the study was to explore the molecular mechanisms characterizing angiolymphatic invasion and therefore identify a gene expression signature related to angiolymphatic invasion.

Methods Gene expression analysis of head and neck squamous cell carcinoma was carried out based on clinical and whole genome expression data provided by The Cancer Genome Atlas. Results were validated in an independent cohort of laryngeal squamous cell carcinoma and confirmed by immunohistochemistry staining.

Results A gene expression signature consisting of six genes (SHH, SLC18A3, LCE3E, LCE2B, LCE3D and DSG-1) related to angiolymphatic invasion was identified. The gene expression profile identified a subset of patients with decreased overall survival $(p=0.02, \log$ rank test), which was most prominent for patients with laryngeal squamous cell carcinoma $(p=0.004$, $\log$ rank test). Furthermore, these patients showed a significant shorter progression-free survival ( $p=0.002, \log$ rank test). By use of this gene expression signature, patients at high risk of recurrence could be identified even if morphological changes were not yet recognizable.

Conclusion Angiolymphatic invasion is characterized by a distinct histopathological phenotype and specific gene expression signature. The newly identified signature might serve as a reliable predictor of outcome in laryngeal cancer and add additional benefit to histopathological evaluation.
\end{abstract}

Keywords Head and neck squamous cell carcinoma $\cdot$ Head and neck cancer · Angiolymphatic invasion · Laryngeal squamous cell carcinoma $\cdot$ HNSCC

Jochen Hess and Dominik Horn contributed equally to this manuscript.

Electronic supplementary material The online version of this article (https://doi.org/10.1007/s00405-020-06214-1) contains supplementary material, which is available to authorized users.

\section{Karl Metzger}

karl.metzger@med.uni-heidelberg.de

1 Department of Oral and Cranio-Maxillofacial Surgery, Heidelberg University Hospital, Im Neuenheimer Feld 400, 69120 Heidelberg, Germany

2 Department of Oral and Cranio-Maxillofacial Surgery, Saarland University Hospital, Kirrberger Str. 100, 66421 Homburg, Germany

3 Department of Otorhinolaryngology, Heidelberg University Hospital, Im Neuenheimer Feld 400, 69120 Heidelberg, Germany

\section{Introduction}

Head and neck squamous cell carcinomas (HNSCC) are affecting more than 600,000 patients per year [1,2]. HNSCC arises from the mucosal lining of the upper aerodigestive

4 Institute of Pathology, Technical University of Munich, TUM School of Medicine, Trogerstr. 18, 81675 Munich, Germany

5 Department of Otorhinolaryngology, Section Experimental and Translational Head and Neck Oncology, Research Group Molecular Mechanisms of Head and Neck Tumors, German Cancer Research Center (DKFZ), Heidelberg University Hospital, Im Neuenheimer Feld 400, 69120 Heidelberg, Germany 
tract, including the oral cavity (OSCC), oropharynx (OPSCC) and larynx (LaSCC). Tobacco smoking, alcohol consumption, betel nut chewing and human papillomavirus (HPV) infection are the main risk factors for the development of HNSCC [3-6]. As a result of multi-modal therapy, 5 -year survival rates have increased up to $67 \%$ for all stages during the last decades $[7,8]$. However, survival rates of laryngeal cancer decreased over the past decades from 66 to $63 \%$ [9], with an unfavorable prognosis for advanced stages $[10,11]$. This is important, as most patients suffering from laryngeal carcinoma present with advanced disease (stage III/IV) at the time of diagnosis [12]. This emphasizes the need for innovations in the field of risk stratification with subsequent therapy adjustments.

Surgical therapy plays a fundamental role in the treatment of HNSCC and is complemented by radiation and chemotherapy in advanced cases (stage III/IV) [8]. Relevant prognostic factors in HNSCC are clinical and histological characteristics, such as tumor size and localization, lymph node extracapsular spread (ECS), and perineural (PNI) and angiolymphatic invasion (ALI) [13-16]. Two of these histological characteristics have been investigated at a molecular level. An 11 gene expression profile for extracapsular spread in oral squamous cell carcinoma serves as a prognosticator of outcome in patients without nodal metastases [17]. In terms of PNI, a gene expression profile revealed a subset of HNSCC at risk of post-surgical recurrence [18]. However, little is known about the molecular mechanisms related to ALI and a molecular signature for ALI has not been identified so far. ALI implies tumorous invasion of lymphatic vessels and intratumoral vascular invasion. It is correlated with higher rates of lymph node metastases and poor prognosis in oral squamous cell carcinoma [19].

The main objective of this study was to explore the molecular mechanisms of ALI and to identify a gene expression signature associated with ALI. Although ALI is a common histopathological characteristic and predictor of outcome in HNSCC, histopathological examination implies a level of uncertainty. It depends on a single pathological examination, and extensive specimens are needed for robust classification. Especially, small biopsy specimens do not meet these demands.

Therefore, a molecular indicator for patients at high risk of recurrence may support histopathological examination, alter therapeutic strategies and improve patient's prognosis. We investigated transcriptome data of HNSCC in relation to the occurrence of ALI and patient's survival based on a global gene expression analysis using TCGA data. We also confirmed newly identified candidate genes in an independent cohort of LaSCC. The whole genome expression data of this cohort were collected in collaboration with the German cancer research center and the Heidelberg Center for Personalized Oncology (DKFZ-HIPO).

\section{Material and methods}

\section{Data acquisition}

\section{TCGA cohort}

A publicly available dataset containing clinical and pathological information on patients with HNSCC was published by the TCGA Research Network. All patients were treated by surgery, followed by radiotherapy or radio-chemotherapy if needed. mRNA count data containing 19,750 genes of 500 patients with HNSCC as well as general clinical and pathological characteristics were downloaded from https://portal.gdc.cancer.gov on January 25,2019 . Updated survival data were retrieved from https://gdc.cancer.gov/about-data/publications/pancanatlas on March 14, 2019. Patients with missing subsite information were excluded from further analysis, resulting in a total of 497 patients.

\section{HIPO-HNC cohort}

Patients with histological confirmed LaSCC $(n=13)$ of the HIPO-HNC cohort were treated at the Heidelberg University Hospital between 2012 and 2016. The seldom expression data of LaSCC were collected in collaboration with the German cancer research center and the Heidelberg Center for Personalized Oncology (DKFZ-HIPO). Patient samples were obtained under the protocol S-206/2011, approved by the Ethics Committee of Heidelberg University, with written informed consent from all participants. This study was conducted in accordance with the Declaration of Helsinki. Sample processing and generation of array-based expression data have been described previously [20]. These data have been uploaded to NCBI's Gene Expression Omnibus and are accessible through GEO Series accession number GSE117973. The clinical and pathological information are summarized in Supplemental table S1. Formalin-fixed paraffin-embedded tissue specimens were retrieved from the tissue bank of the National Center for Tumor Diseases, Heidelberg, Germany.

\section{Data analysis}

\section{Differential gene expression (DGE)}

The DGE analysis was performed on the TCGA cohort in $\mathrm{R}$ version 3.5.1 using the packages DESeq2 and limma $[21,22]$. Genes with an FDR less than 0.05 and a $\log 2-$ fold-change greater 1.5 were considered as significantly 
differentially expressed. FDR values were adjusted using Benjamini-Hochberg correction. The results of DESeq2 and limma were compared, and consistent genes were used for further analysis.

\section{Unsupervised hierarchical clustering}

Transcript data of selected genes were used for unsupervised hierarchical clustering and to construct a heatmap via ClustVis, a web tool for visualizing multivariate data. Hierarchical clustering was performed using Pearson correlation [23].

\section{Chi-squared test and univariate and multivariate analysis}

Chi-squared test was used for comparisons of categorical data and a $p$ value $<0.05$ was considered as statistically significant. Univariate and multivariate analysis was carried out using the Cox regression model. All analyses were performed in $\mathrm{R}$ version 3.5.1.

\section{Survival analysis}

Overall and progression-free survival was estimated using the Kaplan-Meier method. Log-rank test was used to compare the differences between groups. $p$ values less than 0.05 were considered statistically significant. Overall survival was defined as a time period from the date of diagnosis to the last date when the patient was known to be alive (censored) or date of death for any reason (uncensored). Progression-free survival was measured from the time of diagnosis to the day of the last follow-up examination in which the patient was progression free (censored), or to the date of local recurrence of the disease or occurrence of regional or distant metastases (uncensored). Survival analysis was performed in $\mathrm{R}$ version 3.5.1 using the packages survminer, survival and ggplot2.

\section{Immunohistochemistry}

Immunohistochemical staining was carried out on $4 \mu \mathrm{m}$-thick sections from FFPE tissue specimens of the HIPO-HNC cohort. IHC staining was done as described previously [24]. GLI2 was detected by mouse monoclonal antibodies (Santa Cruz Biotechnology, Dallas, USA, clone sc-271786, 1:100 in PBS) and DSG1 was detected by mouse monoclonal antibody (Progen, clone 18107; 1:10 in PBS). The analysis was carried out by two independent observers.

\section{Results}

\section{Association between ALI and progression free or overall survival}

A histological ALI status was available for 336 out of 497 (68\%) patients of the TCGA-HNSC cohort. The impact of ALI on survival was assessed by Kaplan-Meier analysis and $\log$ rank test to confirm a statistically significant shorter progression-free ( $p=0.018, \log$ rank test) and overall survival ( $p=0.008, \log$ rank test) for cases with ALI as compared to ALI-negative counterparts (Fig. 1a, b). The subgroup analysis demonstrated that the association between ALI and recurrent disease or overall survival was evident for LaSCC (PFS: $p=0.047$, OS: $p=0.036, \log$ rank test) and OSCC (PFS: $p=0.021$, OS: $p=0.02, \log$ rank test), but not for OPSCC (PFS: $p=0.98$, OS: $p=0.61, \log$ rank test) (Suppl. Fig. S1A-F).

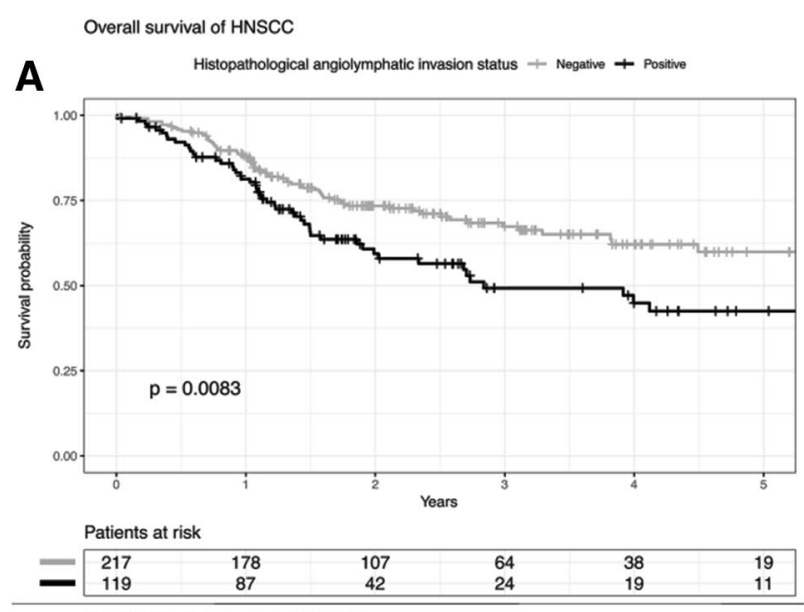

B

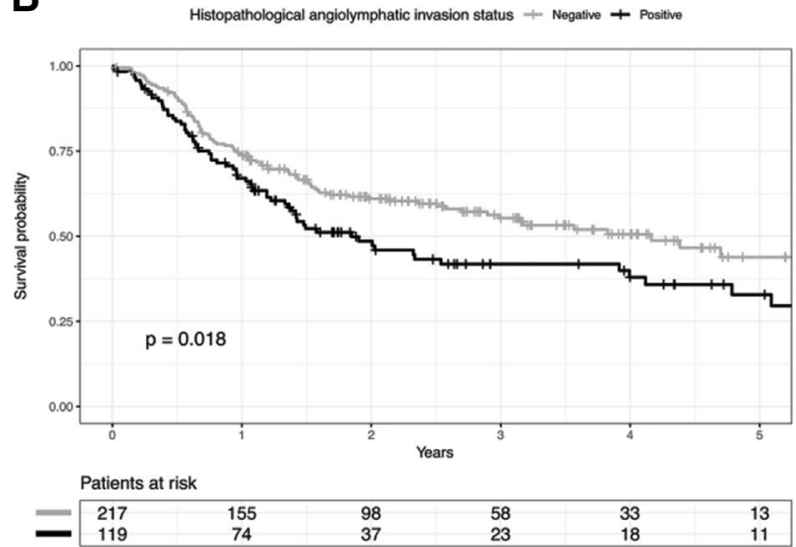

Fig. 1 a Overall survival of patients in the TCGA-HNSCC cohort according to angiolymphatic invasion status. b Progression-free survival of patients in the TCGA-HNSCC cohort according to angiolymphatic invasion status 


\section{ALI-related gene expression signature}

A differential gene expression analysis was performed for subgroups with positive and negative histopathological ALI status $(n=336)$ and significant candidate genes (FDR $<0.05$ and $-1.5<\log 2$-fold-change $>1.5$ ) were considered for further analysis. 93 genes were identified by the DESeq2 package (60 with increased mRNA expression level, 33 with decreased mRNA expression level), and 17 genes were identified by the limma package (two with increased mRNA expression level, 15 with decreased mRNA expression level). To reduce the number of genes and to identify robust candidates, consistent candidate genes were selected. In total, six differentially expressed genes ( $S H H$ and $S L C 18 A 3$ with increased mRNA expression level; $L C E 3 E, L C E 2 B$, $L C E 3 D$ and $D S G-1$ with decreased mRNA expression level) were identified by both packages and selected for further analysis (Suppl. tables S2-3).

We hypothesized that the transcript levels of the six candidate genes are sufficient to predict the risk of ALI in all patients of the TCGA cohort $(n=497)$. Indeed, unsupervised hierarchical clustering using Pearson correlation revealed two main clusters (Suppl. Fig. S2). While tumors in cluster A (264 out of 497; 53\%) were enriched for a negative ALI status, most tumors in cluster B (233 out of 497; 47\%) had a positive ALI status. These data demonstrated the potential of the six-gene expression signature to stratify tumors reliably by ALI status ( $p=2.451 \mathrm{e}-09$, Chi-squared test, Suppl. tables 4-5). Furthermore, previously unclassified patients $(n=161)$ could be categorized to either cluster A or cluster B by use of the presented gene signature. Therefore, the six-gene expression signature enabled segregation of cases for which histological information on the ALI status was not available.

\section{ALI-related gene expression signature predicts tumor recurrence and unfavorable survival in laryngeal carcinoma}

As the six-gene signature stratifies HNSCC reliably according to the ALI status, we addressed the question whether patients in clusters A and B differ in prognosis. All patients of the TCGA-HNSCC $(n=497)$ cohort were considered, including both patients with and without available information on the histological ALI status. Kaplan-Meier plots revealed a significant shorter overall survival for patients in cluster B $(p=0.02, \log$ rank test) (Fig. 2a, b; Supplemental tables 6-7). The subgroup analysis considering distinct subsites of HNSCC demonstrated that differences in overall survival were most prominent for patients with $\operatorname{LaSCC}(p=0.004, \log$ rank test).

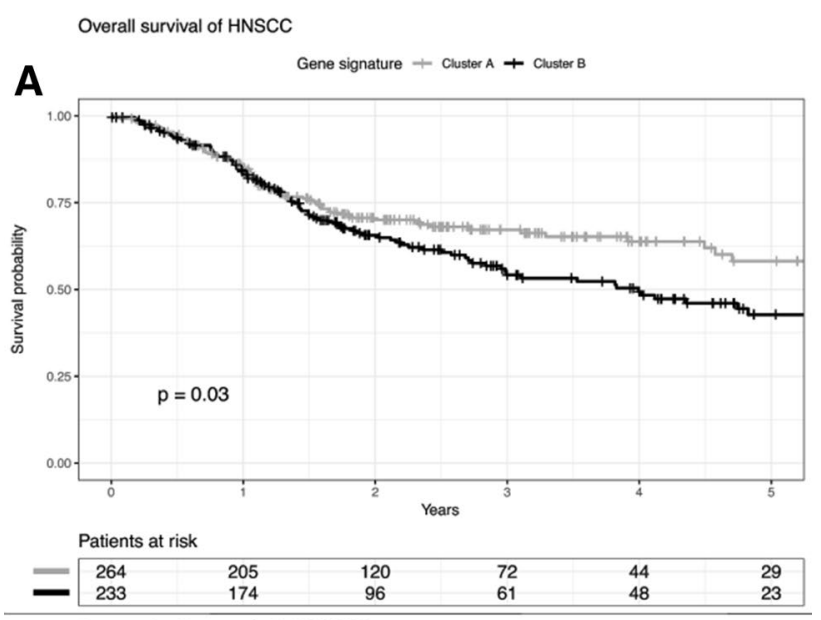

B

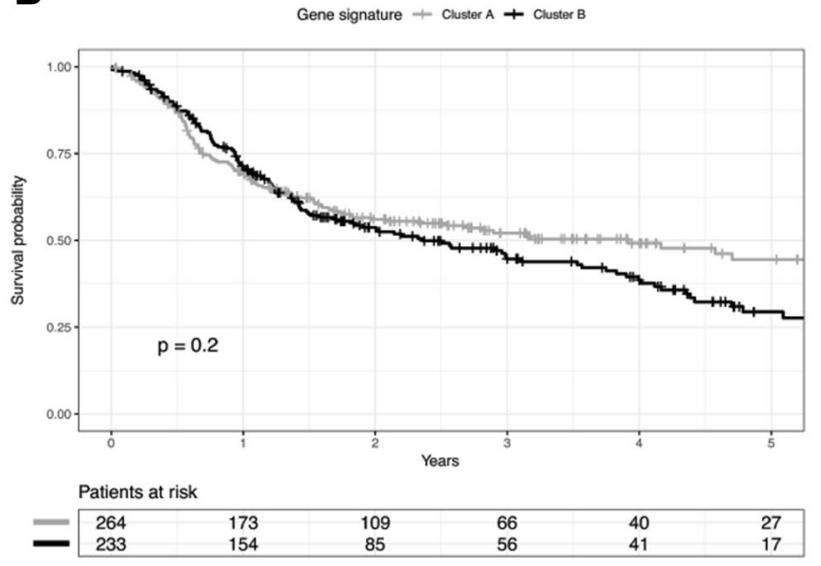

Fig. 2 a Overall survival of patients in the TCGA-HNSCC cohort according to gene expression signature. $\mathbf{b}$ Progression-free survival of patients in the TCGA-HNSCC cohort according to gene expression signature

Moreover, LaSCC patients in cluster B shared a significant shorter progression-free survival ( $p=0.002, \log$ rank test) (Fig. 3a, b, Supplemental table 8-9). Interestingly, 20 out of $41(49 \%)$ LaSCC patients with a negative ALI status as determined by histological inspection were part of cluster $\mathrm{B}$ according to the six-gene expression signature. These patients showed unfavorable PFS (Fig. 4a, b), indicating a substantial amount of patients at risk with a lacking histomorphologic ALI-like phenotype. In contrast, only 4 out of $34(12 \%)$ LaSCC patients with a histologically positive ALI status were part of cluster A according to the six-gene signature (Supplemental table 10).

In conclusion, the newly identified expression signature consisting of six genes is closely associated with ALI and stratifies HNSCC patients at a higher risk for unfavorable progression-free and overall survival, in particular for the subgroup of LaSCC patients, even in histologically unclassified patients. 


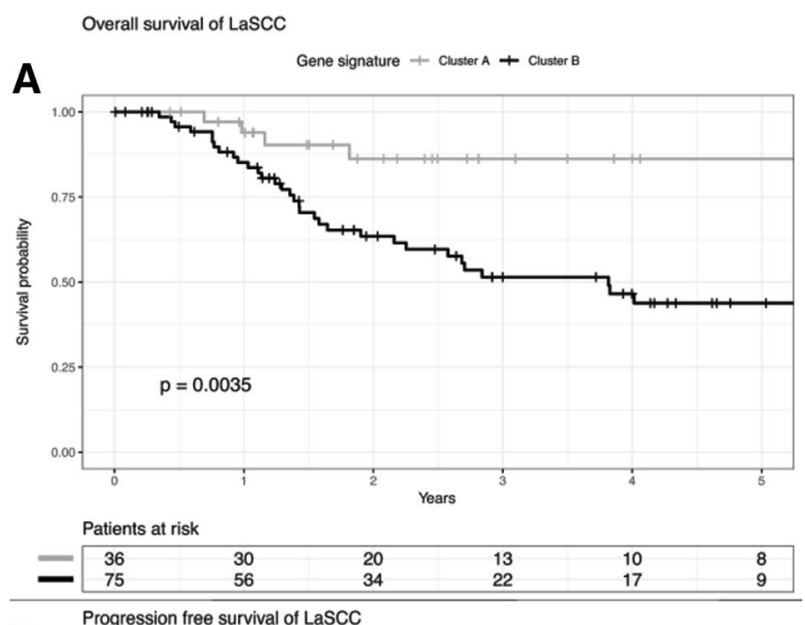

B

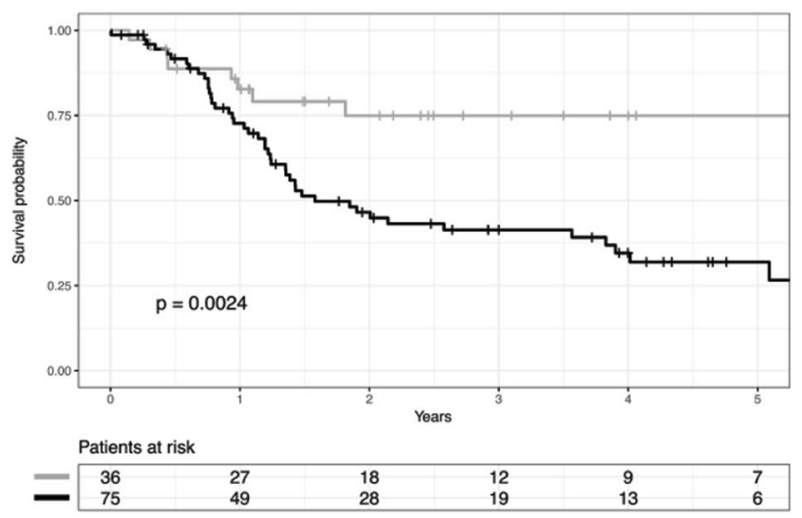

Fig. 3 a Overall survival of patients in the TCGA-LaSCC cohort according to gene expression signature. b Progression-free survival of patients in the TCGA-LaSCC cohort according to gene expression signature

\section{Univariate and multivariate analysis of laryngeal subsite}

Univariate analysis and multivariate Cox regression models were conducted to identify independent risk factors regarding overall and progression-free survival in patients with LaSCC (Table 1). Cluster B, females, positive resection margins, extracapsular spread and perineural invasion were parameters significantly associated with unfavorable OS and PFS in the univariate analysis. In addition, a positive ALI status and non-smoking status were significantly associated with poor OS. ALI status was excluded from the multivariate analysis as it is not independent from the presented gene expression signature. Extracapsular spread and perineural invasion were excluded from the multivariate analysis due to missing information in a large proportion of patients. Non-smoking status (HR 0.11, $p<0.001$ ), females (HR $3.47, p=0.010$ ) and positive resection margins (HR 5.64, $p<0.001$ ) were associated with poor OS in multivariate
Overall survival of ALI negative LaSCC

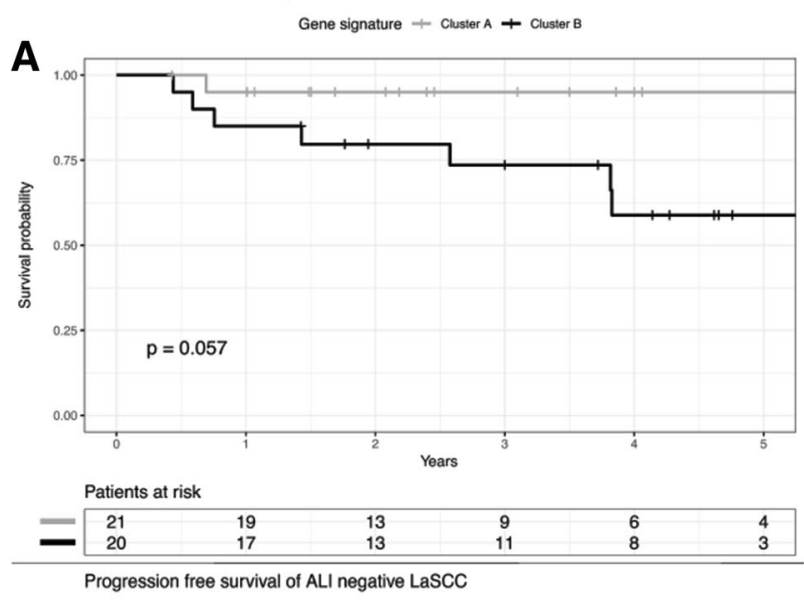

B

Gene signature + Cluster A + Cluster B

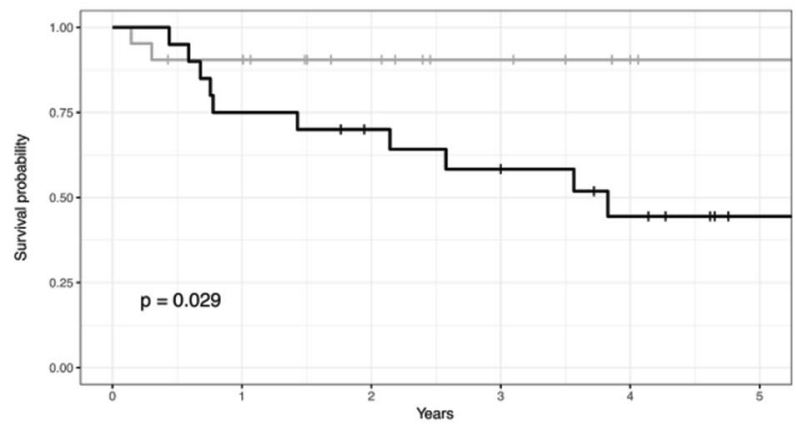

Patients at risk
-\begin{tabular}{|llllll|}
21 & 18 & 12 & 8 & 5 & 3 \\
20 & 15 & 12 & 9 & 6 & 1 \\
\hline
\end{tabular}

Fig. 4 a Overall survival of patients with confirmed negative histopathological ALI status in the TCGA-LaSCC cohort according to gene expression signature. b Progression-free survival of patients with confirmed negative histopathological ALI status in the TCGALaSCC cohort according to gene expression signature

analysis. Cluster B (HR 2.5, $p=0.016)$ and positive resection margins (HR 2.46, $p=0.026$ ) were the only parameters associated with unfavorable PFS in multivariate analysis.

\section{Validation in an independent LaSCC cohort}

The independent validation cohort consisted of 13 patients with LaSCC, who were treated between 2012 and 2016 at the University Hospital Heidelberg (HIPO-HNC). Eleven patients $(85 \%)$ were male and two patients were female (15\%). Patients' age ranged from 49 to 86 years with a median age of 61 years. All patients had a positive smoking history. 6 out of 13 (46\%) patients reported alcohol consumption. Histopathological ALI status was positive in 3 out of $13(23 \%)$ patients (Supplemental table 11). For all tumors, global gene expression data were available and used to conduct unsupervised hierarchical clustering based on the sixgene expression signature. Again, the six-gene expression 
Table 1 Univariate and multivariate analysis of overall and progression-free survival of $\operatorname{LaSCC}(n=111)$

\begin{tabular}{|c|c|c|c|c|c|c|}
\hline \multirow[t]{2}{*}{ Variable } & \multicolumn{3}{|c|}{ Univariate analysis } & \multicolumn{3}{|c|}{ Multivariate analysis } \\
\hline & HR & CI & $p$ value & HR & CI & $p$ value \\
\hline \multicolumn{7}{|l|}{ OS } \\
\hline Tobacco yes vs. no & 0.2242 & $0.0752-0.6685$ & 0.00731 & 0.1123 & $0.03323-0.3791$ & 0.000429 \\
\hline Alcohol & 0.8485 & $0.4516-1.594$ & 0.61 & & & \\
\hline Cluster B vs. A & 3.168 & $1.403-7.155$ & 0.00552 & 2.3603 & $0.94778-5.8778$ & 0.065073 \\
\hline Age & 1.017 & $0.9794-1.057$ & 0.376 & & & \\
\hline Gender $\mathrm{f}$ vs $\mathrm{m}$ & 3.211 & $1.54-6.696$ & 0.00187 & 3.4690 & 1.33880-8.9885 & 0.010449 \\
\hline R1 vs R0 & 4.579 & $1.998-10.49$ & 0.000324 & 5.6384 & 2.2861-13.9066 & 0.000173 \\
\hline Grade & 1.004 & $0.6897-1.462$ & 0.983 & & & \\
\hline $\mathrm{ALI}+\mathrm{vs}-$ & 2.279 & $1.031-5.039$ & 0.0418 & & & \\
\hline $\mathrm{ECS}+\mathrm{vs}-$ & 2.454 & $1.147-5.248$ & 0.0207 & & & \\
\hline $\mathrm{PNI}+\mathrm{vs}-$ & 3.78 & $1.568-9.108$ & 0.00305 & & & \\
\hline Stage III/IV vs I/II & 0.7174 & $0.2476-2.079$ & 0.541 & & & \\
\hline \multicolumn{7}{|l|}{$P F S$} \\
\hline Tobacco yes vs. no & 0.5149 & $0.1823-1.455$ & 0.21 & & & \\
\hline Alcohol yes vs. no & 0.8569 & $0.498-1.474$ & 0.577 & & & \\
\hline Cluster B vs. A & 2.795 & $1.4-5.582$ & 0.00357 & 2.499 & $1.1874-5.261$ & 0.0159 \\
\hline Age & 0.9913 & $0.961-1.023$ & 0.582 & & & \\
\hline Gender $\mathrm{f}$ vs $\mathrm{m}$ & 2.252 & $1.172-4.326$ & 0.0148 & 2.107 & $0.9592-4.627$ & 0.0634 \\
\hline R1 vs R0 & 2.91 & $1.325-6.392$ & 0.00781 & 2.455 & $1.1113-5.423$ & 0.0264 \\
\hline Grade & 1.015 & $0.7184-1.435$ & 0.931 & & & \\
\hline ALI & 2.063 & $0.9957-4.275$ & 0.0514 & & & \\
\hline $\mathrm{ECS}+\mathrm{vs}-$ & 2.397 & $1.141-5.036$ & 0.021 & & & \\
\hline $\mathrm{PNI}+\mathrm{vs}-$ & 3.118 & $1.446-6.722$ & 0.00372 & & & \\
\hline Stage III/IV vs I/II & 1.016 & $0.3974-2.596$ & 0.974 & & & \\
\hline
\end{tabular}

Variables identified as independent prognostic factors by multivariate analysis are highlighted $H R$ hazard ratio, $C I 95 \%$ confidence interval signature revealed two clusters, and all cases with a positive ALI status based on histological examination were part of cluster B (Suppl. Figure 3). Four out of ten patients with a histologically negative ALI status were part of cluster B, indicating specimens lacking histomorphological ALI-like phenotype. However, due to the low number of patients, no survival analysis was conducted for the HIPO-HNC cohort.

\section{Immunohistochemical detection of GLI2 and DSG1}

To confirm altered expression of selected candidate genes of the six-gene expression signature on protein level, GLI2 and DSG-1 protein expression was detected by immunohistochemical staining on tumor sections of the HIPO-LaSCC cohort. GLI2 is well established as a downstream target of $\mathrm{SHH}$ and was used as a surrogate marker for active $\mathrm{SHH}$ signaling. IHC staining confirmed high GLI2, but low DSG1 protein expression in cancer cells of LaSCC attributed to cluster B and an opposite staining pattern for cases in cluster A (Suppl. Figure 4).

In conclusion, the newly identified gene expression signature is associated with histological ALI status in patients with HNSCC and segregates both patients with and without available histological ALI status. It serves as a prognosticator of poor OS in HNSCC and predicts short progressionfree and overall survival in LaSCC. In addition, patients at risk with lacking ALI-like phenotype could be identified. Findings could be confirmed in an independent cohort of LaSCC.

\section{Discussion}

The purpose of this study was to identify a specific gene expression signature associated with ALI in HNSCC. Therefore, we analyzed publicly available data of the TCGAHNSCC cohort. Microscopically determined ALI was associated with a significant shorter progression-free and overall survival in the investigated cohort. This is consistent with current evidence as ALI is a known prognosticator of outcome and patients with ALI-positive HNSCC are recommended to undergo adjuvant radiotherapy [25, 26]. The conducted gene expression analysis unveiled a six-gene expression signature, which was closely related to ALI status and 
predicted tumor recurrence as well as unfavorable survival in HNSCC, in particular for the subsite of LaSCC. Previous studies reported gene expression signatures in association with perineural invasion and extracapsular spread in different subpopulations of HNSCC $[17,18]$. To our knowledge, this is the first study on an ALI-related gene expression signature in the field of HNSCC.

With regard to clinical relevance and in line with the close association to ALI, the newly identified six-gene expression signature served as a risk factor for unfavorable prognosis. Out of the TCGA-HNSCC cohort, 233 patients (47\%) could be identified at a significantly higher risk of mortality. Moreover, the application of this gene signature on subsites of HNSCC revealed patients with LaSCC at a high risk of recurrence and poor overall survival. It is important to emphasize that patients showed recurrence in the presence of this gene signature even in ALI-negative tumors, according to pathological assessment. In addition, patients lacking histological ALI status were classified to either cluster A or cluster B by the presented gene signature. This underlines the additional benefit of the presented signature, as some specimens lack an ALI-like phenotype, while patients experience unfavorable survival.

Pathological examination includes examination of extensive and heterogeneous specimens in patients treated by tumor resection. Traditional risk stratification of patients treated by primary radio(chemo)therapy is challenging, as only small biopsy specimens are available for pathological examination. By use of the presented six-gene signature, biopsy specimen could be analyzed for further characterization of this subgroup of patients. In addition, molecular profiling of circulating tumor cells may be useful in the course of liquid biopsies in the future.

The identified six-gene expression signature includes two genes (SHH, SLC18A3) with increased mRNA expression levels and four genes (LCE3E, LCE2B, LCE3D, DSG1) with decreased mRNA expression levels, suggesting a gain in SHH signaling accompanied by a loss of epithelial differentiation is one underlying principle of ALI. The activation of hedgehog signaling has been described as a prognostic indicator in oral squamous cell carcinoma [27]. An increase in SHH signaling results in cellular proliferation and transformation and it has been described as an indirect angiogenic factor [28, 29]. Angiogenesis as a consequence of SHH function results in robust neovascularization by the induction of an array of angiogenic growth factors, including all isoforms of VEGF [28]. High lymphatic vessel density is correlated with poor OS and lymph node metastasis in HNSCC [30, 31].

SLC18A3 encodes a vesicular acetylcholine transporter (VAChT). The presence of VAChT in epidermal melanocytes and keratinocytes has been demonstrated [32]. Vesamicol, an VAChT antagonist, induces potent apoptosis in bronchioalveolar carcinoma [33]. As cholinergic signaling is upregulated in SCC and blocking cholinergic signaling limits growth of squamous cell lung carcinoma, cholinergic signaling is a potential target in the treatment of HNSCC [34].

All genes in the signature with an inverse correlation to ALI are linked to epithelial differentiation. As a member of desmosomal cadherins, desmoglein-1 (DSG1) is important in epithelial differentiation and intercellular junction mediating a strong cell-cell adhesion [35]. A loss of DSG1 is associated with an invasive phenotype, metastasis and decreased survival in squamous cell carcinoma [36-40].

We confirmed high GLI2, but low DSG1 protein expression in tumors of cluster B by immunohistochemical staining, which was enriched for ALI-positive cases. The presence of a different expression pattern on protein level could support standard histological examination to determine the risk for ALI.

Our results have some limitations. We relied on publicly available data which are subject to multiple errors, including data collection in multiple centers, pathologic examination by different pathologists and recall bias. We are aware of a limited number of patients in our in-house cohort. However, we could not identify a larger cohort of LaSCC containing all six genes of the described gene signature. Moreover, the availability of tissue specimen is a huge advantage of this cohort. While we showed that both low expression levels of genes affecting epidermal differentiation and high expression level of SHH are related to ALI, further studies may clarify whether SHH affects transcription of these epidermal markers.

The presented gene expression signature identifies histological ALI-negative patients at risk of disease progression and poor overall survival. This gene expression signature may serve as a molecular surrogate for ALI, as it reliably stratifies patients by ALI status. Furthermore, it enables risk stratification of patients lacking ALI status and predicts outcome in cases with missing ALI-like phenotype. Hereby, adjuvant therapy might be escalated or de-escalated according to the presented signature.

\section{Conclusion}

We identified a novel gene expression signature related to ALI status in HNSCC patients. This gene expression signature identifies HNSCC patients at risk of poor overall survival and predicts tumor recurrence and unfavorable survival in laryngeal carcinoma, even if histological ALI status was negative or unknown. However, as our independent validation cohort is small, further validation in a large prospective cohort is a major objective for further 
studies. In the future, implementation of additional stratification tools could assist in decision making in adjuvant therapy resulting in better prognosis of patients in need.

Acknowledgements The results published here are partially based upon data generated by the TCGA Research Network: https://www. cancer.gov/tcga. We thank the tissue bank of the National Center for Tumor Disease (Institute of Pathology, University Hospital Heidelberg, Germany) for providing paraffin-embedded tumor specimens. This work was supported by the German Cancer Research CenterHeidelberg Center for Personalized Oncology (DKFZ-HIPO).

Funding Open Access funding enabled and organized by Projekt DEAL.

\section{Compliance with ethical standards}

Conflict of interest The authors declare that they have no conflict of interest.

Ethics approval This study was performed in line with the principles of the Declaration of Helsinki. Approval was granted by the Ethics Committee of Heidelberg University under the protocol S-206/2011.

Consent to participate Informed consent was obtained from all individual participants included in the study.

Open Access This article is licensed under a Creative Commons Attribution 4.0 International License, which permits use, sharing, adaptation, distribution and reproduction in any medium or format, as long as you give appropriate credit to the original author(s) and the source, provide a link to the Creative Commons licence, and indicate if changes were made. The images or other third party material in this article are included in the article's Creative Commons licence, unless indicated otherwise in a credit line to the material. If material is not included in the article's Creative Commons licence and your intended use is not permitted by statutory regulation or exceeds the permitted use, you will need to obtain permission directly from the copyright holder. To view a copy of this licence, visit http://creativecommons.org/licenses/by/4.0/.

\section{References}

1. Bray F, Ferlay J, Soerjomataram I et al (2018) Global cancer statistics 2018: GLOBOCAN estimates of incidence and mortality worldwide for 36 cancers in 185 countries. CA Cancer J Clin 68:394-424. https://doi.org/10.3322/caac. 21492

2. Siegel RL, Miller KD, Jemal A (2018) Cancer statistics, 2018. CA Cancer J Clin 68:7-30. https://doi.org/10.3322/caac.21442

3. Hashibe M, Brennan P, Benhamou S et al (2007) Alcohol drinking in never users of tobacco, cigarette smoking in never drinkers, and the risk of head and neck cancer: pooled analysis in the International Head and Neck Cancer Epidemiology Consortium. J Natl Cancer Inst 99:777-789. https://doi.org/10.1093/jnci/djk179

4. Pelucchi C, Gallus S, Garavello W et al (2006) Cancer risk associated with alcohol and tobacco use: focus on upper aero-digestive tract and liver. Alcohol Res Health 29:193-198

5. Sturgis EM, Cinciripini PM (2007) Trends in head and neck cancer incidence in relation to smoking prevalence: an emerging epidemic of human papillomavirus-associated cancers? Cancer 110:1429-1435. https://doi.org/10.1002/cncr.22963
6. Goldenberg D, Lee J, Koch WM et al (2004) Habitual risk factors for head and neck cancer. Otolaryngol Head Neck Surg 131:986993. https://doi.org/10.1016/j.otohns.2004.02.035

7. Jemal A, Ward EM, Johnson CJ et al (2017) Annual report to the nation on the status of cancer, 1975-2014, featuring survival. J Natl Cancer Inst. https://doi.org/10.1093/jnci/djx030

8. Forastiere A, Koch W, Trotti A, Sidransky D (2001) Head and neck cancer. N Engl J Med 345:1890-1900. https://doi.org/10. 1056/NEJMra001375

9. Hoffman HT, Porter K, Karnell LH et al (2006) Laryngeal cancer in the United States: changes in demographics, patterns of care, and survival. Laryngoscope 116:1-13. https://doi.org/10.1097/01. mlg.0000236095.97947.26

10. Warner L, Chudasama J, Kelly CG et al (2014) Radiotherapy versus open surgery versus endolaryngeal surgery (with or without laser) for early laryngeal squamous cell cancer. Cochrane Database Syst Rev. https://doi.org/10.1002/14651858.CD002027.pub2

11. Forastiere AA, Zhang Q, Weber RS et al (2013) Long-term results of RTOG 91-11: a comparison of three nonsurgical treatment strategies to preserve the larynx in patients with locally advanced larynx cancer. J Clin Oncol 31:845-852. https://doi.org/10.1200/ JCO.2012.43.6097

12. Steuer CE, El-Deiry M, Parks JR et al (2017) An update on larynx cancer. CA Cancer J Clin 67:31-50. https://doi.org/10.3322/caac. 21386

13. Jimenez L, Jayakar SK, Ow TJ, Segall JE (2015) Mechanisms of invasion in head and neck cancer. Arch Pathol Lab Med 139:1334-1348. https://doi.org/10.5858/arpa.2014-0498-RA

14. Bur AM, Lin A, Weinstein GS (2016) Adjuvant radiotherapy for early head and neck squamous cell carcinoma with perineural invasion: a systematic review. Head Neck 38(Suppl 1):E2350 E2357. https://doi.org/10.1002/hed.24295

15. Jardim JF, Francisco AL, Gondak R et al (2015) Prognostic impact of perineural invasion and lymphovascular invasion in advanced stage oral squamous cell carcinoma. Int J Oral Maxillofac Surg 44:23-28. https://doi.org/10.1016/j.ijom.2014.10.006

16. AWMF D (2019) Leitlinienprogramm Onkologie (Deutsche Krebsgesellschaft, Deutsche Krebshilfe, AWMF): Diagnostik, Therapie und Nachsorge des Larynxkarzinoms, Langversion 1.0, 2019, AWMF-Registernummer: 017/076OL, https://www.leitlinienprogr amm-onkologie.de/leitlinien/larynxkarzinom/

17. Wang W, Lim WK, Leong HS et al (2015) An eleven gene molecular signature for extra-capsular spread in oral squamous cell carcinoma serves as a prognosticator of outcome in patients without nodal metastases. Oral Oncol 51:355-362. https://doi.org/ 10.1016/j.oraloncology.2014.12.012

18. Saidak Z, Clatot F, Chatelain D, Galmiche A (2018) A gene expression profile associated with perineural invasion identifies a subset of HNSCC at risk of post-surgical recurrence. Oral Oncol 86:53-60. https://doi.org/10.1016/j.oraloncology.2018.09.005

19. Michikawa C, Uzawa N, Kayamori K et al (2012) Clinical significance of lymphatic and blood vessel invasion in oral tongue squamous cell carcinomas. Oral Oncol 48:320-324. https://doi. org/10.1016/j.oraloncology.2011.11.014

20. Schmitt K, Molfenter B, Laureano NK et al (2019) Somatic mutations and promotor methylation of the ryanodine receptor 2 is a common event in the pathogenesis of head and neck cancer. Int $\mathbf{J}$ Cancer. https://doi.org/10.1002/ijc.32481

21. Love MI, Huber W, Anders S (2014) Moderated estimation of fold change and dispersion for RNA-seq data with DESeq2. Genome Biol 15:550. https://doi.org/10.1186/s13059-014-0550-8

22. Ritchie ME, Phipson B, Wu D et al (2015) limma powers differential expression analyses for RNA-sequencing and microarray studies. Nucleic Acids Res 43:e47. https://doi.org/10.1093/nar/ gkv007 
23. Metsalu T, Vilo J (2015) ClustVis: a web tool for visualizing clustering of multivariate data using principal component analysis and heatmap. Nucleic Acids Res 43:W566-W570. https://doi.org/10. 1093/nar/gkv468

24. Moratin J, Metzger K, Safaltin A et al (2019) Upregulation of PD-L1 and PD-L2 in neck node metastases of head and neck squamous cell carcinoma. Head Neck. https://doi.org/10.1002/ hed. 25713

25. Wolff KD (2012) German national guideline for diagnostik and therapy of OSCC. AWMF-Registry (007-100OL), https://www. leitlinienprogramm-onkologie.de/fileadmin/user_upload/Downl oads/Leitlinien/Mundhoehlenkarzinom/S3-Mundhoehlenkarz inom-OL-Langversion_.pdf. Accessed 18 Nov 2019

26. Adelstein D, Gillison ML, Pfister DG et al (2017) NCCN guidelines insights: head and neck cancers, version 2.2017. J Natl Compr Canc Netw 15:761-770. https://doi.org/10.6004/jnccn. 2017.0101

27. Wang Y-F, Chang C-J, Lin C-P et al (2012) Expression of hedgehog signaling molecules as a prognostic indicator of oral squamous cell carcinoma. Head Neck 34:1556-1561. https://doi.org/ 10.1002/hed.21958

28. Pola R, Ling LE, Silver M et al (2001) The morphogen sonic hedgehog is an indirect angiogenic agent upregulating two families of angiogenic growth factors. Nat Med 7:706-711. https://doi. org/10.1038/89083

29. Kanda S, Mochizuki Y, Suematsu T et al (2003) Sonic hedgehog induces capillary morphogenesis by endothelial cells through phosphoinositide 3-kinase. J Biol Chem 278:8244-8249. https:// doi.org/10.1074/jbc.M210635200

30. Yu M, Liu L, Liang $C$ et al (2014) Intratumoral vessel density as prognostic factors in head and neck squamous cell carcinoma: a meta-analysis of literature. Head Neck 36:596-602. https://doi. org/10.1002/hed.23301

31. Frech S, Hörmann K, Riedel F, Götte K (2009) Lymphatic vessel density in correlation to lymph node metastasis in head and neck squamous cell carcinoma. Anticancer Res 29:1675-1679

32. Elwary SMA, Chavan B, Schallreuter KU (2006) The vesicular acetylcholine transporter is present in melanocytes and keratinocytes in the human epidermis. J Investig Dermatol 126:1879-1884. https://doi.org/10.1038/sj.jid.5700268

33. Lau JK, Brown KC, Thornhill BA et al (2013) Inhibition of cholinergic signaling causes apoptosis in human bronchioalveolar carcinoma. Cancer Res 73:1328-1339. https://doi.org/10.1158/ 0008-5472.CAN-12-3190

34. Song P, Sekhon HS, Fu XW et al (2008) Activated cholinergic signaling provides a target in squamous cell lung carcinoma. Cancer Res 68:4693-4700. https://doi.org/10.1158/0008-5472. CAN-08-0183

35. Kottke MD, Delva E, Kowalczyk AP (2006) The desmosome: cell science lessons from human diseases. J Cell Sci 119:797-806. https://doi.org/10.1242/jcs.02888

36. Bosch FX, Andl C, Abel U, Kartenbeck J (2005) E-cadherin is a selective and strongly dominant prognostic factor in squamous cell carcinoma: a comparison of E-cadherin with desmosomal components. Int J Cancer 114:779-790. https://doi.org/10.1002/ ijc. 20782

37. Chidgey M, Dawson C (2007) Desmosomes: a role in cancer? $\mathrm{Br}$ J Cancer 96:1783-1787. https://doi.org/10.1038/sj.bjc.6603808

38. Shinohara M, Hiraki A, Ikebe T et al (1998) Immunohistochemical study of desmosomes in oral squamous cell carcinoma: correlation with cytokeratin and E-cadherin staining, and with tumour behaviour. J Pathol 184:369-381. https://doi.org/10.1002/(SICI) 1096-9896(199804)184:4<369:AID-PATH1236>3.0.CO;2-L

39. Depondt J, Shabana AH, Florescu-Zorila S et al (1999) Downregulation of desmosomal molecules in oral and pharyngeal squamous cell carcinomas as a marker for tumour growth and distant metastasis. Eur J Oral Sci 107:183-193

40. Krunic AL, Garrod DR, Madani S et al (1998) Immunohistochemical staining for desmogleins 1 and 2 in keratinocytic neoplasms with squamous phenotype: actinic keratosis, keratoacanthoma and squamous cell carcinoma of the skin. Br J Cancer 77:1275-1279

Publisher's Note Springer Nature remains neutral with regard to jurisdictional claims in published maps and institutional affiliations. 